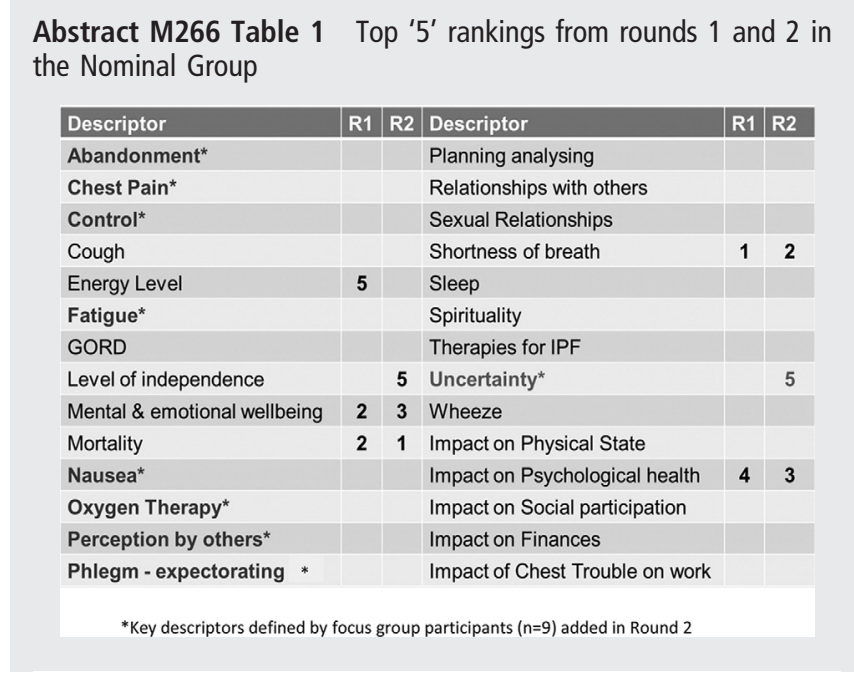

Discussion This methodological approach to item generation will enhance the content validity of the IPF-PROM instrument. Items generated to date will be modified further by 80 patients from 4 UK centres and 20 ILD physicians participating in 3 rounds of a Qualtrics Delphi survey. This study is ongoing. 1 http://www.fda.gov/downloads/Drugs/Guidances/UCM193282.pdf

\section{M267 HEALTH STATUS AND QUALITY OF LIFE IN IDIOPATHIC PULMONARY FIBROSIS AND SARCOIDOSIS: EFFECT OF FATIGUE}

CP Atkins, D Gilbert, C Brockwell, S Robinson, AM Wilson. Norfolk and Norwich University Hospital, Norwich, Norfolk

\subsection{6/thoraxjnl-2014-206260.448}

Introduction and Objective Sarcoidosis and Idiopathic Pulmonary Fibrosis (IPF) are two common forms of interstitial lung disease. Sarcoidosis frequently causes extra-pulmonary disease whereas IPF specifically affects the lungs. Fatigue is a common feature of sarcoidosis, but an association between fatigue and IPF has not been investigated. We investigated the frequency and severity of fatigue in sarcoidosis and IPF, how it correlates with quality of life (QOL) scores, and whether fatigue is affected by disease severity.

Methods This was a cross-sectional questionnaire study of patients with sarcoidosis and IPF. Questionnaire data was analysed to investigate health status, QOL, and symptom prevalence (fatigue, depression and sleepiness). Comparison of scores between groups, and an analysis of the effect of markers of disease severity on fatigue, was undertaken.

Results Questionnaires were administered to 235 participants; 82 healthy volunteers, 76 sarcoidosis patients and 77 IPF patients. IPF patients had statistically higher St George's Respiratory Questionnaire $(p=0.034)$ and Epworth Sleepiness Scale scores $(p=0.003)$ than sarcoidosis patients, but there was no difference in mean fatigue scores. When stratified by questionnaire scores (Table 1), including pathological fatigue levels, no statistical difference was seen between IPF and sarcoidosis, although there was a trend towards a higher frequency of 'severe fatigue' in sarcoidosis. Fatigue scores correlated strongly with quality of life scores (King's Brief Interstitial Lung Disease score and St George's Respiratory Questionnaire) in both IPF ( $r=-$
0.615 and 0.659 respectively) and sarcoidosis $(r=-0.529$ and 0.502). In sarcoidosis, no measures of dyspnoea or disease severity (spirometry abnormality, immunosuppression use or extrapulmonary disease) were associated with fatigue scores. In IPF increasing dyspnoea scores were associated with increased fatigue scores $(\mathrm{p}<0.001)$.

Conclusions Both sarcoidosis and IPF patients suffer with high levels of fatigue, although the sarcoidosis cohort showed a trend towards greater frequency of severe fatigue compared with IPF. In IPF patients increasing fatigue was associated with worsening dyspnoea, suggesting an association with disease progression, but no similar relationship was seen in sarcoidosis. This suggests that fatigue in sarcoidosis occurs independently of common markers of disease activity, whereas it occurs as a sequelae of progressive disease in IPF.

\section{Abstract M267 Table 1 Prevalence of Anxiety, depression, sleepiness and fatigue symptoms}

\begin{tabular}{|c|c|c|c|c|}
\hline & & $\begin{array}{l}\text { Healthy } \\
\text { Volunteers }\end{array}$ & Sarcoidosis & IPF \\
\hline \multirow{4}{*}{$\begin{array}{l}\text { HADS-A } \\
\text { Anxiety }\end{array}$} & \begin{tabular}{|l|} 
Normal $(\%)$ \\
\end{tabular} & $73(89.0)$ & $42(60.9)$ & $54(71.4)$ \\
\hline & Mild (\%) & $5(6.1)$ & $18(26.1)$ & $14(18.2)$ \\
\hline & Moderate (\%) & $3(36.6)$ & $8(11.6)$ & $6(7.8)$ \\
\hline & Severe (\%) & $1(1.2)$ & $1(1.4)$ & $2(2.6)$ \\
\hline \multirow{4}{*}{$\begin{array}{l}\text { HADS-D } \\
\text { Depression }\end{array}$} & Normal (\%) & $78(95.1)$ & $51(73.9)$ & $63(82.9)$ \\
\hline & Mild (\%) & $3(36.6)$ & $10(14.5)$ & $3(3.9)$ \\
\hline & Moderate (\%) & $1(1.2)$ & $5(7.2)$ & $8(10.6)$ \\
\hline & Severe (\%) & $0(0)$ & $3(4.3)$ & $2(2.6)$ \\
\hline \multirow{3}{*}{$\begin{array}{l}\text { Epworth } \\
\text { Sleepiness } \\
\text { Scale }\end{array}$} & $\begin{array}{l}\text { No sleepiness } \\
\text { (\%) }\end{array}$ & $66(85.7)$ & $47(67.1)$ & $52(85.2)$ \\
\hline & Borderline (\%) & $7(9.1)$ & $19(27.1)$ & $7(11.5)$ \\
\hline & $\begin{array}{l}\text { Severe } \\
\text { Sleepiness (\%) }\end{array}$ & $4(5.2)$ & $4(5.7)$ & $2(3.3)$ \\
\hline \multirow{3}{*}{$\begin{array}{l}\text { Fatigue } \\
\text { Assessment } \\
\text { Scale }\end{array}$} & Normal (\%) & $74(91.4)$ & $34(49.3)$ & $40(54.8)$ \\
\hline & Fatigued (\%) & $5(6.2)$ & $27(39.1)$ & $30(41.1)$ \\
\hline & $\begin{array}{l}\text { Severe Fatigue } \\
\text { (\%) }\end{array}$ & $2(2.4)$ & $8(11.6)$ & $3(4.1)$ \\
\hline
\end{tabular}

\section{M268 THE IPF DIAGNOSIS - COMMUNICATING A LIFE SENTENCE}

${ }^{1} \mathrm{~S}$ Wibberley, ${ }^{2} \mathrm{Y}$ Ochiai, ${ }^{2} \mathrm{R}$ Pitt, ${ }^{2} \mathrm{~N}$ Mathieson. ${ }^{1}$ British Lung Foundation, London, UK; ${ }^{2}$ Boehringer Ingelheim Ltd UK, Bracknell, Berkshire, UK

\subsection{6/thoraxjnl-2014-206260.449}

Background The aim was to explore the patients' emotional experience of receiving a diagnosis of IPF.

Methods Market research was conducted with an independent agency. Patients with IPF were asked to record a personal account of their experience on a hand-held camera. Face to face interviews with patients were conducted in their home. Carers were also interviewed to add an alternative perspective.

Results The sample included 13 male and 3 female patients with IPF. Patients with lung function impairment of all severities were included, five patients were treated with oxygen therapy and another had received a lung transplant. There was a national spread geographically throughout England.

There is a fine balance to providing information and patients can benefit from an individually tailored approach. Too much information at the start can be overwhelming. Too little information can leave the individual uncertain about how to deal with their future.

Gaps that were identified focused on the practicalities of living with IPF, including social care. There was a high expectation for their physician to explain the trigger for developing IPF. Patients felt a blame culture exists, whereby others felt that IPF is self-inflicted, like COPD, particularly when a patient was 
taking oxygen therapy. Patients may be left with feelings of anger at missed opportunities and concern for lost years of intervention and appropriate palliative care support.

Conclusions The diagnosis of IPF is a devastating one, which can be challenging to manage. Carers, patient groups and expert support at diagnosis were found to be invaluable to patients during this time.

\section{M269 THE EMOTIONAL TURMOIL OF IPF}

${ }^{1} S$ Wibberley, ${ }^{2} Y$ Ochiai, ${ }^{2} \mathrm{R}$ Pitt, ${ }^{2} \mathrm{~N}$ Mathieson. ${ }^{1}$ British Lung Foundation, London, UK; ${ }^{2}$ Boehringer Ingelheim Ltd UK, Bracknell, Berkshire, UK

\subsection{6/thoraxjnl-2014-206260.450}

Background Our aim was to understand the emotions patients experience in IPF, from initial symptoms to IPF specialist management.

Methods Market research was conducted with an independent agency. Patients with IPF were asked to record a personal account of their experience on a hand-held camera. Face to face interviews with patients were conducted in their home. Carers were also interviewed to add an alternative perspective.

Results The sample included 13 male and 3 female patients with IPF. Patients with lung function impairment of all severities were included, five patients were treated with oxygen therapy and another had received a lung transplant.

Many patients had a very active lifestyle before developing IPF, leading to a high degree of frustration with the limitations imposed on their physical ability. A protracted time to diagnosis of a rare lung disease while symptoms progressed often led to distrust with their primary healthcare physician. Lack of expert knowledge about the condition often resulted in variable handling of the situation, with patients often finding themselves involved in a type of 'role-reversal' whereby they informed their primary healthcare physician about their own condition.

IPF specialists were perceived as their "guardian angels". Despite being given a terminal diagnosis, patients felt reassured that they were receiving appropriate management for their condition. This stemmed from the perception that specialists treating them had appropriate knowledge and a feeling they were supported by the specialist team.

Conclusions As with other rare diseases, patients appear to gain most reassurance from HCP's with a clear understanding of their condition. This highlights the benefit of expert multidisciplinary teams for IPF.

\section{M270 OBTAINING INFORMATION WHEN YOU HAVE A RARE DISEASE - THE POTENTIAL FOR IPF SUPPORT GROUPS}

${ }^{1} \mathrm{~S}$ Wibberley, ${ }^{2} \mathrm{Y}$ Ochiai, ${ }^{2} \mathrm{R}$ Pitt, ${ }^{2} \mathrm{~N}$ Mathieson. ${ }^{1}$ British Lung Foundation, London, UK; ${ }^{2}$ Boehringer Ingelheim Ltd UK, Bracknell, Berkshire, UK

10.1136/thoraxjnl-2014-206260.451

Background The aim was to explore the ways in which patients with IPF obtain information about their condition.

Methods Market research was conducted with an independent agency. Patients with IPF were asked to record a personal account of their experience on a hand-held camera. Face to face interviews with patients were conducted in their home. Carers were also interviewed to add an alternative perspective.
Results The sample included 13 male and 3 female patients with IPF. Patients with lung function impairment of all severities were included, five patients were treated with oxygen therapy and another had received a lung transplant.

Patients reported finding information from a variety of sources, including primary healthcare professionals, patient information leaflets, the internet, district nurses and support groups. Most valued sources of information were IPF physicians, nurse specialists and patient support groups.

Gaps identified by patients were the need for high quality information including, 1) accurate and complete information about IPF, 2) clarity on the difficulty of predicting life expectancy, 3) how to access services and benefits, 4) how palliative care can help, 5) why support groups are beneficial, 6) how to modify lifestyle as capabilities change, 7) how to live and travel with oxygen and 8) how to explain oxygen to others.

Conclusions Support groups are under-developed, with great potential to help patients and their carers. Support groups are well placed to provide advice for everyday living that the healthcare community may be unable to offer. There is also a need to improve the standard of written information currently available for patients with IPF.

\section{M271 A SURVEY OF TRAINEE EXPERIENCES IN INTERSTITIAL LUNG DISEASE}

${ }^{1} \mathrm{C}$ Sharp, ${ }^{2} \mathrm{M}$ Gibbons. ${ }^{1}$ North Bristol NHS Trust, Bristol, UK; ${ }^{2}$ Royal Devon and Exeter NHS Foundation Trust, Exeter, UK

\subsection{6/thoraxjnl-2014-206260.452}

Interstitial lung disease (ILD) is a major area of respiratory medicine It is important that trainees gain competence and confidence in this area.

Methods A survey of BTS trainee members was conducted in November 2013 to examine training provision in ILD, including trainee's opportunities, experience and confidence in aspects of ILD.

Results There were 104 respondents out of a possible 574 .

$33 \%$ of respondents were not expecting any subspecialty clinics in ILD in the course of their training. $42 \%$ of trainees expect to spend 3 months or less attending specialist clinics. Trainee attendance at MDTs is far from guaranteed, with $45 \%$ expecting to attend less than half during their period in these hospitals.

The majority of trainees are trained in performing BAL for cell differential analysis (73\%) and transbronchial biopsies (84\%), however only $48 \%$ are confident performing transbronchial biopsies. Confidence interpreting investigation results increases with the frequency these are performed.

The self rated knowledge in a range of subject areas was also assessed and demonstrated that most areas were moderately well understood, however knowledge of the less frequently encountered IIPs was rated lower.

$54 \%$ of trainees felt their ILD training was inadequate for SCE preparation. 94\% would value a BTS Short Course on ILD to improve their knowledge and confidence.

Discussion This survey highlights areas where there are clear opportunities to enhance the training of registrars in ILDs. It is worth noting that some of the data is in conflict with previous BTS surveys in this area and there is the possibility of self-selection bias in the response population.

Whilst most trainees are trained in performing relevant procedures, their confidence interpreting the results of common investigations in ILD is low. To give evidence of training and 